\title{
Skin and mucosal manifestations of cancer treatments in Lomé (Togo): A series of 35 cases
}

\section{Abla Sefako Akakpo', Ablavi Ahoefa Oyenitiniwa Adani-lfè², Julienne Noude Téclessou ${ }^{3}$, Panawe Kassang $^{1}$, Abas Mouhari-Toure ${ }^{4}$, Koussake Kombaté ${ }^{3}$, Bayaki Saka ${ }^{1}$, Palokinam Pitché ${ }^{1}$}

${ }^{\text {}}$ Service de dermatologie et IST, CHU Sylvanus Olympio, Université de Lomé, Togo, ${ }^{2}$ Service d'oncologie médicale, CHU Sylvanus Olympio, Université de Lomé, Togo, Service de dermatologie et IST, CHU Campus, Université de Lomé, Togo, ${ }^{3}$ Service de dermatologie et IST, CHU Kara, Université de Kara, Togo

Corresponding author: Abla Sefako Akakpo, MD, E-mail: sefasyl@yahoo.fr

\begin{abstract}
Objective: This study aimed to describe the mucocutaneous manifestations of anti-cancer treatments in cancer patients in Lomé (Togo). Patients and Method: This is a descriptive cross-sectional study conducted in the oncology department of the Sylvanus Olympio University Hospital and in a private medical clinic in Lomé over 6 months. Results: A total of 35 patients were included with 26 patients (74.3\%) receiving either mono or multidrug therapy or $9(25.7 \%)$ receiving radiotherapy. The mean age of the patients was $52 \pm 11.36$ years with extremes ranging from 24 to 71 years and the sex ratio $(\mathrm{M} / \mathrm{F})$ was 0.35 . The main drugs used during chemotherapy were platinum salts $(n=18 ; 69.2 \%)$, antimetabolites $(n=15 ; 61.5 \%)$, taxanes $(n=9 ; 34.6)$, topoisomerase inhibitors $(n=7 ; 26.9 \%)$, alkylating agents $(n=6 ; 23.1 \%)$. The main mucocutaneous manifestations observed were localized hyperpigmentation (palms and soles, fingertips, tongue) noted in 11 patients $(n=42.3 \%)$, followed by alopecia observed from the first treatment $(n=8 ; 30.8 \%)$, skin xerosis $(n=4$; $15.4 \%)$, and oral involvement (aphthoid lesions, gingivitis), $(n=4 ; 15.4 \%)$. Radiodermatitis grade 3, 2 and 1 was noted in 4 patients (44; 4\%), 4 other patients (44.4\%) and 1 patient (11.2\%) respectively. Conclusion: Our study identifies localized hyperpigmentation, alopecia, grade 3 and 2 radiodermatitis as the respective mucocutaneous manifestations of multidrug therapy and radiotherapy in Lomé, Togo.
\end{abstract}

Key words: mucocutaneous lesions, chemotherapy, radiotherapy, Togo

How to cite this article: Akakpo AS, Ablavi Adani-Ifè AO, Téclessou JN, Kassang P, Mouhari-Toure A, Kombaté K, Saka B, Pitché P. Skin and mucosal manifestations of cancer treatments in Lomé (Togo): A series of 35 cases. Our Dermatol Online. 2022;13(Supp. 1):7-14.

Submission: 24.01.2022; Acceptance: 01.02.2022

DOI:10.7241/ourd.2022S1.2

(C) Our Dermatol Online Supp. 1. 2022 


\section{Manifestations cutanéo-muqueuses des traitements anticancéreux à Lomé (Togo): Une série de $35 \mathrm{ca}$}

\section{Abla Sefako Akakpo', Ablavi Ahoefa Oyenitiniwa Adani-Ifè2, Julienne Noude Téclessou³, Panawe Kassang', Abas Mouhari-Toure ${ }^{4}$, Koussake Kombaté3, Bayaki Saka', Palokinam Pitché1}

${ }^{1}$ Service de dermatologie et IST, CHU Sylvanus Olympio, Université de Lomé, Togo, ${ }^{2}$ Service d'oncologie médicale, CHU Sylvanus Olympio, Université de Lomé, Togo, Service de dermatologie et IST, CHU Campus, Université de Lomé, Togo, ${ }^{3}$ Service de dermatologie et IST, CHU Kara, Université de Kara, Togo

Corresponding author: Abla Sefako Akakpo, MD, E-mail: sefasyl@yahoo.fr

\section{RÉSUMÉ}

Objectif: Le but de cette étude était de décrire les manifestations cutanéomuqueuses des traitements anticancéreux chez les patients atteints de cancers à Lomé (Togo). Patients et méthodes: Il s'agit d'une étude transversale descriptive, menée dans le service d'oncologie du centre hospitalier universitaire Sylvanus Olympio et dans un cabinet médical privé de Lomé pendant une période de 6 mois. Résultats: Au total 35 patients ont été inclus avec 26 patients $(74,3 \%)$ qui ont reçu soit une mono ou une polychimiothérapie et $9(25,7 \%)$ une radiothérapie. Lâge moyen des patients était de $52 \pm 11,36$ ans avec des extrêmes allant de 24 à 71 ans et le sex-ratio $(\mathrm{H} / \mathrm{F})$ était de 0,35 . Les principaux médicaments utilisés au cours de la chimiothérapie étaient les sels de platines $(n=18 ; 69,2 \%)$, les antimétabolites $(n=15 ; 61,5 \%)$, les taxanes $(n=9$; $34,6)$, les inhibiteurs de topoisomérases $(n=7 ; 26,9 \%)$ et les agents alkylants $(n=6 ; 23,1 \%)$. Les principales manifestations cutanéo-muqueuses observées étaient une hyperpigmentation localisée (paumes et plantes, bout des doigts, langue) notée chez 11 patients $(n=42,3 \%)$, suivie d'une alopécie observée dès la première cure $(n=8 ; 30,8 \%)$, une xérose cutanée $(n=4$; $15,4 \%)$ et une atteinte buccale (lésions aphtoïdes, gingivite), $(n=4 ; 15,4 \%)$. Une radiodermite de grade 3,2 et 1 était notée respectivement chez 4 patientes $(44 ; 4 \%), 4$ autres patientes $(44,4 \%)$ et 1 patiente $(11,2 \%)$. Conclusion: Notre étude identifie l'hyperpigmentation localisée, l'alopécie, les radiodermites de grade 3 et 2 comme étant respectivement les manifestations cutanéo-muqueuses de la polychimiothérapie et la radiothérapie à Lomé (Togo).

Mots clés: lésions cutanéo-muqueuses, chimiothérapie, radiothérapie, Togo

\section{INTRODUCTION}

Au cours des deux dernières décennies, des avancées significatives ont été réalisées dans le domaine de la biologie des thérapies du cancer avec de nombreuses nouvelles molécules. Ces nouveaux traitements ont profondément modifié la prise en charge et le pronostic de certains cancers [1,2]. Les manifestations cutanéo-muqueuses font partie des toxicités les plus couramment observées de ces traitements [2,3]. Les principales complications cutanéo-muqueuses de la chimiothérapie sont maintenant bien individualisées. Elles sont d'ailleurs volontiers assez spécifiques de ces traitements anticancéreux, notamment l'effluvium anagène, la mucite, le syndrome mains-pieds [4]. La toxicité de la chimiothérapie est dose-dépendante et en lien avec l'association des produits [1].

Par ailleurs, les rayonnements ionisants au cours de la radiothérapie à l'instar de la chimiothérapie, sont aussi utilisés pour traiter diverses formes de cancers. Aux Etats-Unis, au Canada, en Europe et en Australie, $50 \%$ des patients cancéreux reçoivent une radiothérapie pendant leur maladie [5]. Parmi ces patients, $95 \%$ développeront une radiodermite qui est l'un des effets secondaires les plus courants [6,7], due à des lésions tissulaires causées par une accumulation d'inflammation, de nécrose et de mort cellulaire. Elle se manifeste par une variété de symptômes incluant érythème, œdème, 
desquamation sèche ou humide, et une douleur allant de légère à sévère ou débilitante [8] .

La prise en charge des différents types de cancers au Togo se faisait par spécialité médicale. Un avis oncologique sur place n'a été rendu possible qu'à partir de 2017, permettant ainsi, l'utilisation de protocoles thérapeutiques variés avec de large gamme de traitements anticancéreux et plus de visibilité sur leur toxicité. A notre connaissance, une seule étude au Togo [9] avait porté sur une molécule anticancéreuse (Imatinib) et avait relevé les hypopigmentations diffuses et les odèmes périorbitaires comme étant ses effets secondaires cutanés dominants. Nous menons donc cette étude afin de décrire les différents effets secondaires cutanéo-muqueux des traitements anticancéreux observés chez les patients atteints de cancer à Lomé.

\section{Patients et méthodes}

Il s'agit d'une étude transversale descriptive, menée dans le service d'oncologie du Centre Hospitalier Universitaire Sylvanus Olympio et dans un cabinet médical privé de Lomé de novembre 2020 à mai 2021. Les patients suivis pour un cancer et ayant débuté leur première séance de chimiothérapie et ceux revenus du Sweden Ghana medical centre d'Accra (le Togo ne disposant pas de radiothérapie que depuis février 2021, les patients nécessitant ce traitement ont été envoyés au Ghana et ont été vus à leur retour) ont été inclus dans cette étude.

Les variables étudiées étaient: les données sociodémographiques (âge, sexe, profession), les antécédents (notion de tabagisme, alcoolisme, diabète, HTA, atopie, séropositivité au VIH), la nature et localisation du cancer, le type histologique du cancer, les traitements anticancéreux (chimiothérapie et modalités, radiothérapie), et les lésions cutanéomuqueuses apparues au cours de la chimiothérapie et de la radiothérapie.

Les chimiothérapies sont données en fonction du type de cancer et selon divers protocoles [10]:

i) ABVD (Doxorubicine $25 \mathrm{mg} / \mathrm{m}^{2}$, Bléomycine $15 \mathrm{mg} / \mathrm{m}^{2}$, Vinblastine $10 \mathrm{mg} / \mathrm{m}^{2}$, Dacarbazine $375 \mathrm{mg} / \mathrm{m}^{2}$, administré tous les 15 jours en intraveineuse.

ii) FEC (5 Fluorouracile $500 \mathrm{mg} / \mathrm{m}^{2}$, Epirubicine $100 \mathrm{mg} / \mathrm{m}^{2}$, Cyclophosphamide $500 \mathrm{mg} / \mathrm{m}^{2}$, administré en intraveineuse tous les 21 jours. iii) EOX: Epirubicine $50 \mathrm{mg} / \mathrm{m}^{2}$, Oxaliplatine $130 \mathrm{mg} / \mathrm{m}^{2}$ en intraveineuse tous les 21 jours, Capécitabine $625 \mathrm{mg} / \mathrm{m}^{2}$ deux fois par jour per os pendant les 21 jours.

iv) XELOX: Capécitabine 625 à $1000 \mathrm{mg} / \mathrm{m}^{2}$ per os deux fois par jour, Oxaliplatine 120 à $130 \mathrm{mg} / \mathrm{m}^{2}$ en intraveineuse tous les 21 jours.

v) FOLFOX: 5 Fluorouracile $400 \mathrm{mg} / \mathrm{m}^{2}$, Leucovorine $400 \mathrm{mg} / \mathrm{m}^{2}$, Oxaliplatine $130 \mathrm{mg} / \mathrm{m}^{2}$, 5Fluorouracile $2400 \mathrm{mg} / \mathrm{m}^{2}$ en perfusion continue (46 heures) administré tous les 14 jours.

vi) MCAVI: Méthotrexate $30 \mathrm{mg} / \mathrm{m}^{2}$, Vinblastine $3 \mathrm{mg} / \mathrm{m}^{2}$ les $1^{\text {er }}, 15^{\mathrm{e}}, 22^{\mathrm{e}}$ jour du cycle et Carboplatine $\mathrm{AUC}_{5}$ le $\mathrm{l}^{\text {er }}$ jour en intraveineuse tous les 28 jours.

vii) 5FU-CDDP: 5 Fluorouracile $1000 \mathrm{mg} / \mathrm{m}^{2} /$ jour en perfusion continue du $\mathrm{l}^{\mathrm{er}}$ au $5^{\mathrm{e}}$ jour et Cisplatine $100 \mathrm{mg} / \mathrm{m}^{2}$ le $1^{\text {er }}$ jour chaque 21 jours.

viii) Docétaxel $100 \mathrm{mg} / \mathrm{m}^{2}$ en intraveineuse tous les 21 jours

ix) Capécitabine $1250 \mathrm{mg} / \mathrm{m}^{2}$ deux fois par jour per os ( ${ }^{\text {er }}$ jour au $14^{\mathrm{e}}$ jour), tous les 21 jours.

x) Paclitaxel $220 \mathrm{mg} / \mathrm{m}^{2}$, Carboplatine $\mathrm{AUC}_{6}$, en intraveineuse tous les 21 jours

xi) Paclitaxel 135 à $175 \mathrm{mg} / \mathrm{m}^{2}$, Carboplatine $\mathrm{AUC}_{5}$, en intraveineuse tous les 21 jours

xii) Gemcitabine $1000 \mathrm{mg} / \mathrm{m}^{2}$, le $1^{\text {er }}$, le $8^{\mathrm{e}}$ jour et le $15^{\text {ème }}$ jour du cycle en intraveineuse tous les 28 jours

La radiothérapie du cancer du sein a été faite sur la paroi thoracique atteinte, la chaîne mammaire interne et la chaîne sus claviculaire à la dose de 50 Grays en 25 fractions, 5 jours par semaine pendant 5 semaines. L'irradiation pour les métastases cérébrales a été faite sur l'encéphale à la dose de 30 Grays en 10 fractions, 5 jours par semaine pendant 2 semaines.

Nous avons utilisé le Common Terminology Criteria for Adverse Events (CTCAE) version 4.03 [11,12] pour la classification de la radiodermite et avons parlé de:

i) Grade 0 si pas de dermatite;

ii) Grade 1 si dermatite légère: érythème léger ou desquamation sèche;

iii) Grade 2 si dermatite modérée: érythème modéré à vif, desquamation humide inégale, principalement aux plis cutanés; œedème modéré;

iv) Grade 3 si dermatite sévère: desquamation humide dans les zones autres que les plis cutanés; saignement induit par traumatisme mineur ou abrasion;

v) Grade 4 si dermatite sévère: peau nécrosée ou ulcération complète du derme, saignement spontané du site impliqué, indication de greffe de peau. 


\section{Ethics Statement}

Le consentement éclairé des patients ont été ont été obtenus et les chefs services ont donné leur pour faire cette étude.

\section{Resultats}

Nous avons recruté 35 patients souffrant de cancer dont l'âge moyen était de 52 11 , 36 ans avec des extrêmes allant de 24 à 71 ans et un sex-ratio de 0,35 . Les patients étaient en majorité des commerçants/vendeurs (20\%), des fonctionnaires $(17,1 \%)$ et des sans-emplois $(17,1 \%)$. La durée d'évolution du cancer avant la consultation oncologique était de 6 à 12 mois dans 42, 8\% des cas (Tableau 1). Le cancer du sein était le plus retrouvé dans 37, 1\% (n=13), (Tableau 1). Chez plus de la moitié des patients $(\mathrm{n}=20,57,1 \%)$, on notait la présence d'adénopathies dont $60 \%$ étaient de siège axillaire $(\mathrm{n}=12)$. Parmi les patients 67, 6\% $(\mathrm{n}=23)$ présentaient des métastases. Les sites métastatiques les plus fréquents étaient le foie $(n=9,39,1 \%)$, le poumon $(n=8,34,8 \%)$ et les ganglions $(n=7,30,4 \%)$. Cinq patients $(21,7 \%)$ avaient présenté une carcinose péritonéale et 4 patients $(17,4 \%)$ avaient des lésions secondaires osseuses.

\begin{tabular}{lcc} 
Tableau 1: Profession, durée d'évolution, type de cancer & \\
\hline & Effectif $\mathbf{n}=35$ & $\%$ \\
\hline Profession & 7 & 20,0 \\
Commerçant/revendeur & 6 & 17,1 \\
Fonctionnaire & 6 & 17,1 \\
Sans emploi & 4 & 11,4 \\
Ménagères & 3 & 8,6 \\
Retraité & 1 & 2,9 \\
Etudiant/étudiante & 8 & 22,9 \\
Autres professions & \\
Durée d'évolution & & \\
<6 mois & 3 & 8,6 \\
6-12 mois & 15 & 42,8 \\
1-5 ans & 14 & 40,00 \\
$>5$ ans & 3 & 8,6 \\
Types de cancer & & \\
Cancer du sein & 13 & 37,1 \\
Cancer gastrique & 4 & 11,4 \\
Cancer du rectum & 3 & 8,5 \\
Métastases cérébrales de cancer de sein & 2 & 5,7 \\
Cancer du col de l'utérus & 2 & 5,7 \\
Cancer de l'endomètre & 2 & 5,7 \\
Cancer du colon & 2 & 5,7 \\
Cancer du poumon & 2 & 5,7 \\
Cancer de l'ovaire & 1 & 2,9 \\
Cancer de la fesse & 1 & 2,9 \\
Léiomyosarcome du bras & 1 & 2,9 \\
Lymphome de Hodgkin & 1 & 2,9 \\
Néoplasie intraépithéliale de la verge & 2,9 \\
\hline Autes profssit: accocheuse, agent debanque & & \\
\hline
\end{tabular}

Autres professions*: accoucheuse, agent de banque, aide-laboratoire, caissière, cordonnier, enseignant, infirmier, ingénieur
Le carcinome canalaire infiltrant $(\mathrm{n}=18 ; 51,3 \%)$, l'adénocarcinome $(n=15 ; 42,9 \%)$, le sarcome $(n=1$, $2,9 \%)$ et le lymphome $(\mathrm{n}=1 ; 2,9 \%)$ étaient les types histologiques de cancer les plus retrouvés.

Selon le type de traitement reçu, 26 patients (74, $3 \%$ ) avaient eu une chimiothérapie et $9(25,7 \%)$ une radiothérapie.

Les principaux médicaments utilisés au cours de la chimiothérapie étaient les sels de platines $(n=18 ; 69$, $2 \%)$, les antimétabolites $(\mathrm{n}=15 ; 61,5 \%)$, les taxanes $(n=9 ; 34,6 \%)$, (Tableau 2$)$. Une polychimiothérapie a été faite chez $24(92,3 \%)$ patients.

Les principales manifestations cutanéo-muqueuses observées étaient une hyperpigmentation localisée (Figs. la et lb), notée chez 11 patients $(n=42,3 \%)$ apparaissant de façon progressive à la $1^{\text {ère }}$ cure $(\mathrm{n}=3$, $11,5 \%), 2^{\text {ème }}(n=6 ; 23,1 \%), 3^{\text {ème }}(n=9 ; 34,6 \%), 4^{\text {ème }}$ $(\mathrm{n}=9 ; 34,6 \%), 5^{\text {ème }}(\mathrm{n}=11 ; 42,3 \%)$ et la $6^{\text {ème }}$ cure $(n=8 ; 30,8 \%)$. Les schémas thérapeutiques étaient le protocole oxaliplatine + capécitabine et le protocole 5 fluorouracile + epirubicine + cyclophosphamide. Elle était prononcée de façon progressive au fur et à mesure des cures et siégeait aux paumes et à la plante des pieds $(n=5 ; 45,5 \%)$, à la plante des pieds $(n=4 ; 36,3 \%)$, au bout des doigts $(n=1 ; 9,1 \%)$, sur la langue $(n=1 ; 9,1 \%)$.

Une alopécie était notée dès la première cure $(n=8$; $30,8 \%$ ) avec l'utilisation des protocoles 5 fluorouracile + épirubicine + cyclophosphamide et paclitaxel +

Tableau 2: Principaux groupes de médicaments utilisés

\begin{tabular}{lcc}
\hline & Effectif $(\mathbf{n}=\mathbf{2 6})$ & $\%$ \\
\hline Sels de platines & 18 & 69,2 \\
Oxaliplatine & 10 & \\
Cisplatine & 1 & \\
Carboplatine & 7 & 61,5 \\
Antimétabolites & 16 & \\
5 fluorouracile & 5 & \\
Capécitabine & 10 & \\
Gemcitabine & 1 & \\
Taxanes & 9 & \\
Paclitaxel ou Taxol & 7 & \\
Docétaxel ou Taxotère & 2 & \\
Inhibiteurs de topoisomérases & 7 & \\
Epirubicine & 6 & \\
Doxorubicine & 1 & \\
Agents alkylants & 6,1 \\
Cyclophosphamide & 5 & 3,8 \\
Dacarbazine & 1 & \\
Agents scindants & 1 & \\
Bleomycine & 1 & \\
Vinca-alcaloïdes & 1 & \\
Vinblastine & 1 & \\
\hline
\end{tabular}


carboplatine. Une atteinte buccale (lésions aphtoïdes et gingivite) avait été retrouvée chez 2 patients (7, $7 \%$ ) après la $1^{\text {ère }}$ cure par utilisation de capécitabine + oxaliplatine; chez 4 patients $(15,4 \%)$ à la $4^{\mathrm{e}}$ cure avec 5 fluorouracile + épirubicine + cyclophosphamide. La seule atteinte unguéale observée était une mélanonychie (Figs. 2a et 2b), notée chez 4 patients à la $4{ }^{\text {ème }}$ cure où étaient utilisés capécitabine + oxaliplatine; doxorubicine + bleomycine + vinblastine + dacarbazine; carboplatine. + paclitaxel; docétaxel. Une seule patiente

Tableau 3 A: Manifestations cutanéo-muqueuses en fonction des périodes de cure et molécules utilisées

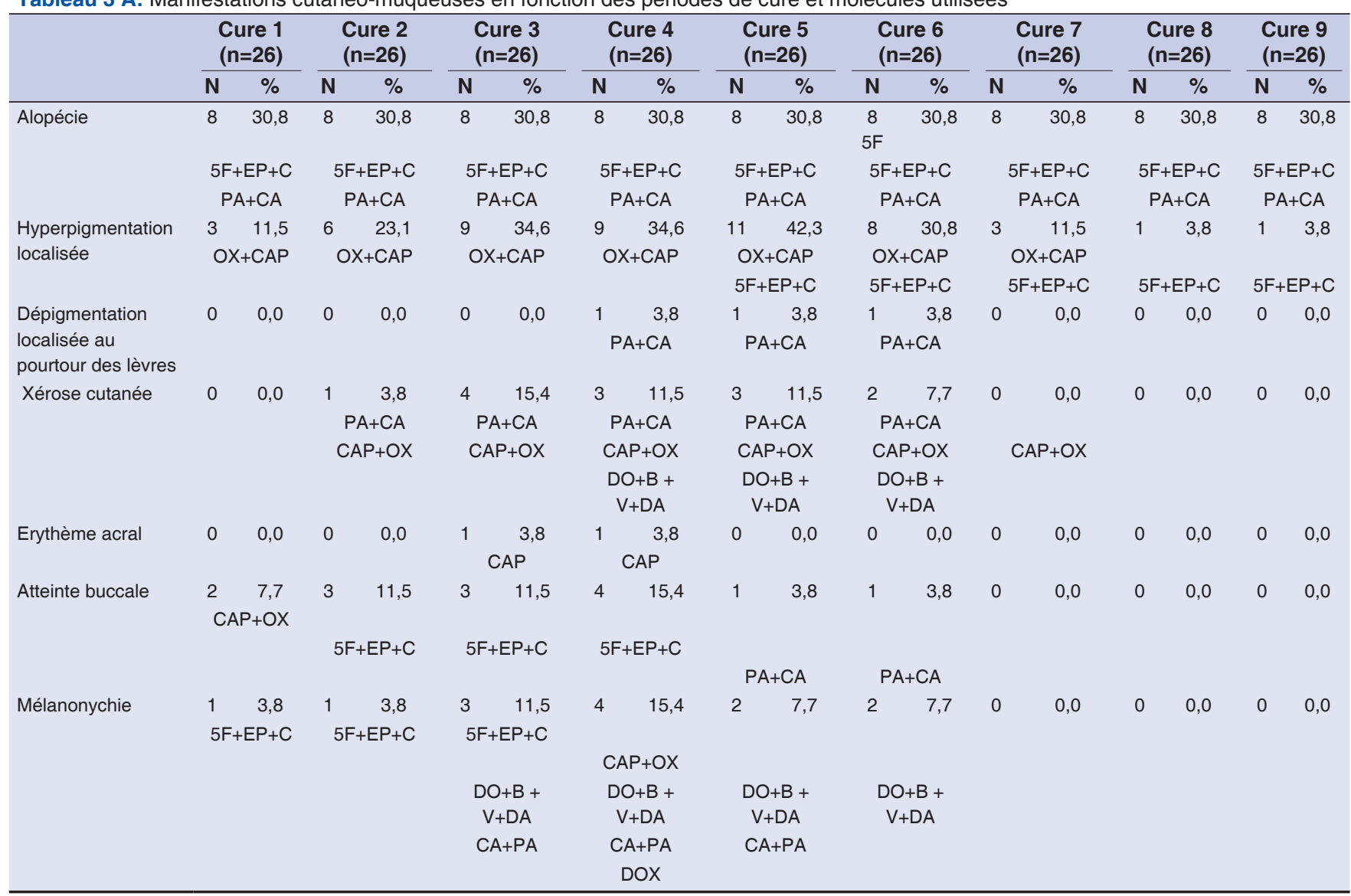

5F=5Fluorouracile, EP=Epirubicine, $\mathrm{C}=$ Cyclophosphamide, $\mathrm{PA}=\mathrm{Paclitaxel}, \mathrm{CA}=$ Carboplatine, OX=Oxaliplatine, $\mathrm{CAP}=\mathrm{Capécitabine,} \mathrm{DO}=\mathrm{Doxorubicine}$, $\mathrm{B}=$ Bleomycine, $\mathrm{DA}=$ Dacarbazine, $\mathrm{V}=$ Vinblastine, $\mathrm{DOX}=$ Docétaxel

Tableau 3 B: Manifestations cutanéo-muqueuses en fonction des périodes de cure et molécules utilisées

\begin{tabular}{|c|c|c|c|c|c|c|c|c|c|c|c|c|c|c|c|c|c|c|}
\hline & \multicolumn{2}{|c|}{$\begin{array}{l}\text { Cure } 1 \\
(n=26)\end{array}$} & \multicolumn{2}{|c|}{$\begin{array}{l}\text { Cure } 2 \\
(n=26)\end{array}$} & \multicolumn{2}{|c|}{$\begin{array}{l}\text { Cure } 3 \\
(n=26)\end{array}$} & \multicolumn{2}{|c|}{$\begin{array}{l}\text { Cure } 4 \\
(n=26)\end{array}$} & \multicolumn{2}{|c|}{$\begin{array}{l}\text { Cure } 5 \\
(n=26)\end{array}$} & \multicolumn{2}{|c|}{$\begin{array}{l}\text { Cure } 6 \\
(n=26)\end{array}$} & \multicolumn{2}{|c|}{$\begin{array}{l}\text { Cure } 7 \\
(n=26)\end{array}$} & \multicolumn{2}{|c|}{$\begin{array}{l}\text { Cure } 8 \\
(n=26)\end{array}$} & \multicolumn{2}{|c|}{$\begin{array}{l}\text { Cure } 9 \\
(n=26)\end{array}$} \\
\hline & $\mathbf{N}$ & $\%$ & $\mathbf{N}$ & $\%$ & $\mathbf{N}$ & $\%$ & $\mathbf{N}$ & $\%$ & $\mathbf{N}$ & $\%$ & $\mathbf{N}$ & $\%$ & $\mathbf{N}$ & $\%$ & $\mathbf{N}$ & $\%$ & $\mathbf{N}$ & $\%$ \\
\hline \multirow[t]{2}{*}{ Veinite } & 0 & 0,0 & 1 & 3,8 & 2 & 7,7 & 1 & 3,8 & 1 & 3,8 & 0 & 0,0 & 0 & 0,0 & 0 & 0,0 & 0 & 0,0 \\
\hline & & & \multicolumn{2}{|c|}{$5 \mathrm{~F}+\mathrm{EP}+\mathrm{C}$} & \multicolumn{2}{|c|}{$5 F+E P+C$} & \multicolumn{2}{|c|}{$5 F+E P+C$} & \multicolumn{2}{|c|}{$5 F+E P+C$} & & & & & & & & \\
\hline \multirow[t]{2}{*}{ kératodermie plantaire } & 0 & 0,0 & 0 & 0,0 & 1 & 3,8 & 1 & 3,8 & 2 & 7,7 & 2 & 7,7 & 0 & 0,0 & 0 & 0,0 & 0 & 0,0 \\
\hline & & & & & \multicolumn{2}{|c|}{$\mathrm{CAP}+\mathrm{OX}$} & \multicolumn{2}{|c|}{$\mathrm{CAP}+\mathrm{OX}$} & \multicolumn{2}{|c|}{$\mathrm{CAP}+\mathrm{OX}$} & \multicolumn{2}{|c|}{$\mathrm{CAP}+\mathrm{OX}$} & & & & & & \\
\hline \multirow[t]{2}{*}{ Lichen cutané } & 0 & 0,0 & 0 & 0,0 & 1 & 3,8 & 1 & 3,8 & 0 & 0,0 & 0 & 0,0 & 0 & 0,0 & 0 & 0,0 & 0 & 0,0 \\
\hline & & & & & \multicolumn{2}{|c|}{$\mathrm{DO}+\mathrm{B}+\mathrm{V}+\mathrm{DA}$} & \multicolumn{2}{|c|}{$\mathrm{DO}+\mathrm{B}+\mathrm{V}+\mathrm{DA}$} & & & & & & & & & & \\
\hline \multirow[t]{2}{*}{ Herpès labial } & 0 & 0,0 & 0 & 0,0 & 0 & 0,0 & 0 & 0,0 & 1 & 3,8 & 0 & 0,0 & 0 & 0,0 & 0 & 0,0 & 0 & 0,0 \\
\hline & & & & & & & & & \multicolumn{2}{|c|}{$5 F+E P+C$} & & & & & & & & \\
\hline \multirow[t]{2}{*}{ Pyodermite de la jambe droite } & 0 & 0,0 & 0 & 0,0 & 0 & 0,0 & 0 & 0,0 & 0 & 0,0 & 1 & 3,8 & 1 & 3,8 & 0 & 0,0 & 0 & 0,0 \\
\hline & & & & & & & & & & & \multicolumn{2}{|c|}{$5 F+E P+C$} & \multicolumn{2}{|c|}{$5 F+E P+C$} & & & & \\
\hline \multirow[t]{2}{*}{ Dermite du bras droit } & 0 & 0,0 & 0 & 0,0 & 0 & 0,0 & 0 & 0,0 & 0 & 0,0 & 0 & 0,0 & 0 & 0,0 & 0 & 0,0 & 1 & 3,8 \\
\hline & & & & & & & & & & & & & & & & & \multicolumn{2}{|c|}{$5 F+E P+C$} \\
\hline \multirow[t]{2}{*}{ Orgelet } & 0 & 0,0 & 0 & 0,0 & 0 & 0,0 & 1 & 3,8 & 0 & 0,0 & 0 & 0,0 & 0 & 0,0 & 0 & 0,0 & 0 & 0,0 \\
\hline & & & & & & & \multicolumn{2}{|c|}{$5 F+E P+C$} & & & & & & & & & & \\
\hline
\end{tabular}

5F=5 Fluorouracile, EP=Epirubicine, $\mathrm{C}=$ Cyclophosphamide, $\mathrm{PA}=\mathrm{Paclitaxel}, \mathrm{CA}=\mathrm{Carboplatine}, \mathrm{OX}=\mathrm{Oxaliplatine}, \mathrm{CAP}=\mathrm{Capé}$ itabine, $\mathrm{DO}=\mathrm{Doxorubicine}$,

$\mathrm{B}=$ Bleomycine, $\mathrm{DA}=$ Dacarbazine, $\mathrm{V}=$ Vinblastine, $\mathrm{DOX}=$ Docétaxel 
avait eu un lichen cutané à partir de sa $3^{\text {ème }}$ cure et était sous doxorubicine + bleomycine + vinblastine + dacarbazine (Tableau 3a et 3b), (Tableau 4).

La durée de la radiothérapie pour le cancer du sein et des métastases cérébrales du cancer du sein était respectivement de 5 semaines et 2 semaines. Les radiodermites de grade 3 sont apparues à la fin des irradiations ( 5 semaines). Une radiodermite de grade 3 (Fig. 3), 2 et 1 était notée respectivement chez 4 patientes $(44 ; 4 \%), 4$ autres patientes $(44,4 \%)$ et 1 patiente $(11,2 \%)$.

\section{DISCUSSION}

Les principales limites de cette étude sont $i$ ) le faible nombre de patients inclus posant le problème de représentativité, ii) la difficulté de rattacher un effet

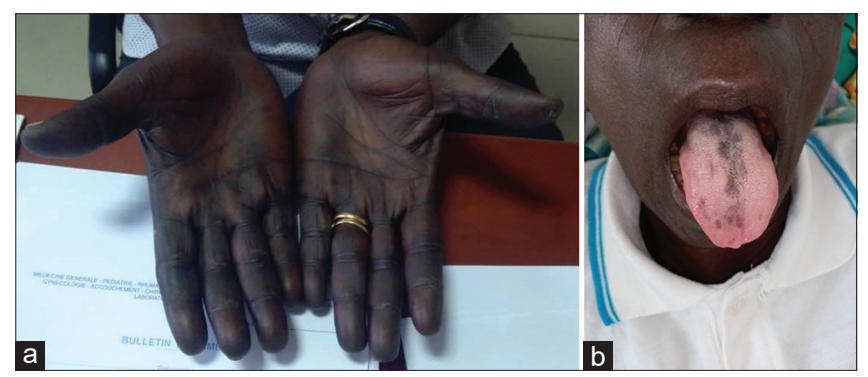

Figure 1: a: Hyperpigmentation localisée aux mains, b: Hyperpigmentation localisée à la langue

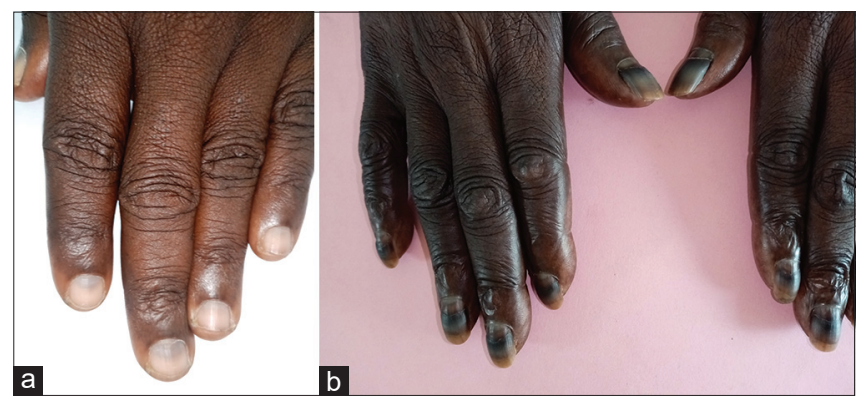

Figure 2 a et b: Mélanonychie

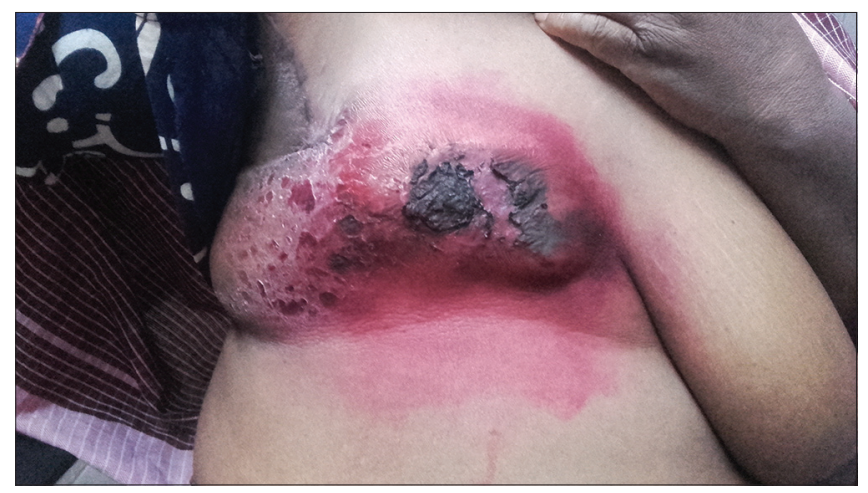

Figure 3: Radiodermite de grade 3 secondaire à un médicament particulier iii) l'évolution des effets secondaires non rapportée dans l'étude soit à cause du décès des patients, soit des perdus de vue. Néanmoins, ce travail nous donne une idée de la fréquence des manifestations cutanéo-muqueuses des chimiothérapies et des radiothérapies à Lomé.

En effet, les thérapies anticancéreuses peuvent entraîner une grande diversité de toxicités dermatologiques affectant la peau, la muqueuse buccale, les cheveux, et les ongles avec une variété de tableaux cliniques, parfois trompeurs. Les dermatologues et les oncologues doivent être conscients de ces effets secondaires afin de pouvoir les reconnaître et les prendre en charge $[13,14]$. Dans notre travail, ces toxicités étaient dominées par une hyperpigmentation localisée $(n=11$; $42,3 \%$ ) évoluant de façon croissante et prononcée avec les protocoles utilisant les sels de platines + antimétabolites; antimétabolites + inhibiteurs de topoisomérases + agents alkylants. Lalopécie était la $2^{\text {ème }}$ toxicité la plus fréquente $(n=8 ; 30,8 \%)$. Elle a été observée dès la première cure avec l'association des antimétabolites + inhibiteurs de topoisomères topoisomérases + agents alkylants et l'association des taxanes + sels de platines.

Lalopécie est l'effet secondaire cutané le plus fréquent et le plus redouté des patients de la chimiothérapie au point que, pour cette seule raison, jusqu'à $14 \%$ de femmes refusent de la faire $[14,15]$. Bien qu'elle puisse survenir avec la plupart des médicaments cytotoxiques, certains présentent un risque plus élevé en terme de fréquence et/ou de gravité, en particulier les agents alkylants (cyclophosphamide et ifosfamide), les anthracyclines (doxorubicine), les taxanes (paclitaxel et docétaxel), les dérivés de la camptotécine (irinotécan et topotécan). Sa sévérité est liée à la dose et à la durée

Tableau 4: Manifestations cutanéo-muqueuses et leur fréquence

\begin{tabular}{lcc} 
& Effectif $(\mathbf{n = 2 6})$ & $\%$ \\
\hline Hyperpigmentation localisée & 11 & 42,3 \\
Alopécie & 8 & 30,8 \\
Xérose cutanée & 4 & 15,4 \\
Atteinte buccale* $^{*}$ & 4 & 15,4 \\
Mélanonychie & 4 & 15,4 \\
Veinite & 2 & 7,7 \\
Kératodermie plantaire & 2 & 7,7 \\
Dépigmentation localisée au Pourtour des lèvres & 1 & 3,8 \\
Erythème acral & 1 & 3,8 \\
Herpès labial & 1 & 3,8 \\
Lichen cutané & 1 & 3,8 \\
Pyodermite de la jambe droite & 1 & 3,8 \\
Dermite du bras droit & 1 & 3,8 \\
Orgelet & 1 & 3,8 \\
\hline
\end{tabular}

Atteinte buccale*: lésions aphtoïdes $(n=2)$, gingivite $(n=2)$ 
du traitement [14]. Les protocoles relatifs au cancer du sein $(37,1 \%$ dans notre étude) incluant souvent des taxanes, sont signalés comme provoquant les alopécies [16].

L'anomalie pigmentaire la plus courante induite par la chimiothérapie est l'hyperpigmentation qui peut affecter la peau, les muqueuses, les cheveux, les ongles [14]. Les tableaux cliniques ne sont pas spécifiques des médicaments reçus. Une même chimiothérapie peut induire plusieurs types cliniques de pigmentation et, inversement, un même aspect clinique peut résulter de traitements distincts. Limputabilité propre à chaque molécule est donc souvent difficile à établir, les schémas thérapeutiques reposant avant tout sur l'utilisation de polychimiothérapie [4]. L'hyperpigmentation des ongles fréquemment observée au cours de la chimiothérapie peut être diffuse (cyclophosphamide ou ifosfamide) ou partielle, présentant un motif longitudinal ou plus rarement horizontal. Les bandes horizontales sont plus rares que les bandes longitudinales. Elle est observée après un traitement aux anthracyclines. Les agents alkylants tels que le cyclophosphamide, l'ifosfamide, et le melphalan sont fréquemment responsables de la pigmentation longitudinale, tandis que l'hydroxyurée donne généralement des bandes pigmentées horizontales et longitudinales [14,17]. Dans notre étude, les 4 cas de mélanonychie ont été retrouvés après utilisation de doxorubicine + bléomycine + vinblastine + dacarbazine, carboplastine + paclitaxel; docétaxel. Ainsi, différents régimes de traitement entraînent différents types de toxicité pour les ongles, allant de la mélanonychie à la leuconychie et à l'onycholyse [13] .

Le développement de lésions pigmentées sur la muqueuse buccale est assez rare. Labsence de symptomatologie et le caractère peu visible de la muqueuse buccale expliquent sans doute que ces lésions passent le plus souvent inaperçues. Les principales molécules inductrices sont le busulfan, la bléomycine, le cyclophosphamide, le cisplatine, le 5 fluorouracile, la doxorubicine, le tégafur, la daunorubicine, l'hydroxyurée et plus récemment la capécitabine. Les lésions peuvent survenir sous polychimiothérapie [4]. Dans notre travail, nous avons eu un cas dû au protocole capécitabine + oxaliplatine

Les autres atteintes de la muqueuse buccale en dehors des troubles pigmentaires sont les saignements, la xérostomie, la glossite, les réactions lichénoïdes, ainsi qu'une hyperkératose et une hyperplasie gingivale. Les taux les plus élevés sont rapportés pour les associations anthracyclines + docétaxel + 5 fluorouracile $(66 \%)$; docétaxel +5 fluorouracile $(46 \%)$ et docétaxel +5 fluorouracile + platine $(43 \%)$ [18]. Dans notre travail, nous avons enregistré des lésions aphtoïdes et une gingivite aux capécitabine + oxaliplastine; 5 fluorouracile + epirubicine + cyclophosphamide.

Le syndrome mains-pieds est un effet secondaire cutané bien établi de certains agents principalement la capécitabine, le 5 fluorouracile, le docétaxel et la doxorubicine. L'érythème, la dysesthésie, la douleur, les craquelures et la desquamation localisés sur les paumes et les plantes sont les symptômes les plus fréquents. Bien qu'il soit un état clinique réversible et ne mettant pas la vie en danger, il affecte la qualité de vie du patient [19]. Dans notre travail, nous avons eu un patient $(3,8 \%)$ qui avait eu un érythème acral sous capécitabine et deux patients $(7,7 \%)$ qui avaient une kératodermie plantaire sous capécitabine + oxaliplatine.

La xérose et le prurit sont des effets secondaires largement sous représentés dans de nombreuses thérapies anticancéreuses, mais il a été démontré qu'ils réduisent la qualité de vie du patient plus que la plupart des autres toxicités. Ils sont causés par l'inhibition du récepteur du facteur de croissance épidermique (EGFR) [13]. Dans notre travail, nous avons retrouvé une xérose cutanée dans $15,4 \%$ de cas $(\mathrm{n}=4)$ due aux protocoles doxorubicine + bléomycine + vinblastine + dacarbazine; paclitaxel + carboplatine; capécitabine + oxaliplatine.

Le cancer du sein est traité par une excision chirurgicale de la tumeur, suivie d'une radiothérapie adjuvante destinée à diminuer le risque de récidive locorégionale et à améliorer la survie [12,20]. Dans notre étude, la radiothérapie $(n=9)$ a été effectuée pour un cancer du sein et des métastases cérébrales de cancer du sein. La radiodermite aigue est l'effet secondaire le plus fréquent [20]. Bien que les lésions tissulaires commencent après la fraction initiale de radiothérapie, les symptômes de la dermite de radiation apparaissent généralement dans les 1 à 4 semaines suivant le début du rayonnement, et restent ou continuent d'augmenter pendant la durée d'exposition. Il a été rapporté que les symptômes guérissent environ 2 à 4 semaines après la fin de la radiothérapie. Les radiodermites peuvent avoir un effet profond sur la qualité de vie [12]. Dans notre travail, les radiodermites de grade 3 sont apparues à la fin des irradiations ( 5 semaines). Lérythème est la première manifestation, survenant chez plus de $90 \%$ de ces patients, suivie d'une desquamation 
humide chez plus de 30\% des patients [21]. L'hypo et l'hyperpigmentation post-inflammatoire sont des changements chroniques courants observés chez les patients, la perte des follicules pileux, des ongles et des glandes sébacées. La télangiectasie et la fibrose sont également fréquentes chez les patients souffrant de radiodermite chronique, cette dernière les prédispose aux ulcères, à la dégradation de la peau, à la rétraction des tissus, à la limitation des mouvements qui en découle, à la douleur et à la thrombose [11].

\section{CONCLUSION}

Notre étude identifie l'hyperpigmentation localisée, l'alopécie, les radiodermites de grade 3 et 2 comme étant respectivement les manifestations cutanéo-muqueuses de la polychimiothérapie et de radiothérapie à Lomé (Togo).

\section{ACKNOWLEDGEMENTS}

Les atteintes cutanées au cours des traitements anticancéreux sont des toxicités fréquentes altérant la qualité de vie des patients.

\section{Statement of Human and Animal Rights}

All the procedures followed were in accordance with the ethical standards of the responsible committee on human experimentation (institutional and national) and with the 2008 revision of the Declaration of Helsinki of 1975.

\section{Statement of Informed Consent}

Informed consent for participation in this study was obtained from all patients.

\section{Références}

1. Bonnot J, Pillon F. Chimiothérapie anticancéreuse et prise en charge bucco-dentaire. Actual Pharm. 2013;522:49-52.

2. Sanmartin O. Skin manifestations of targeted antineoplastic therapy. Curr Probl Dermatol. 2018;53:93-104.

3. Kubicka-Wolkowska J, Kedzierska M, Lisik-Habib M, Potemski P. Skin toxicity in a patient with ovarian cancer treated with pegylated liposomal doxorubicin: a case report and review of the literature. Oncol Lett. 2016;12:5332-34.

4. Sibaud V, Fricain JC, Baran R, Robert C. Anomalies pigmentaires induites par les traitements anticancéreux. Première partie: les chimiothérapies. Ann Dermatol. 2013;140:183-96.

5. Siegel R, DeSantis C, Virgo K, Stein K, Mariotto A, Smith T, et al. Cancer treatment and survivorship statistics, 2012. CA Cancer J Clin. 2012;62:220-41.

6. McQuestion M. Evidence-based skin care management in radiation therapy: clinical update. Semin Oncol Nurs. 2011;27:e1-17.

7. Ryan JL. Ionizing radiation: the good, the bad, and the ugly. J Invest Dermatol. 2012;132:985-93.

8. Drost L, Li N, Vesprini D, Sangha A, Lee J, Leung E, et al. Prospective study of breat radiation dermatitis. Clin Breast Cancer. 2018;18:e789-95.

9. Teclessou JN, Kueviakoe IM, Akakpo AS, Kombate K, Saka B, Mouhari-Toure A, et al. Effets secondaires cutanés de l'imatinib: étude portant sur 36 patients. J. Rech. Sci. Univ. Lomé (Togo). 2017;19:453-56.

10. Vignot S. Principaux protocoles de chimiothérapie en oncologie. Paris: DDL éditions, 2010. p257.

11. Singh M, Alavi A, Wong R, Akita S. Radiodermatitis: a review of our current understanding. Am J Clin Dermatol. 2016;17:277-92.

12. Feight D, Baney T, Bruce S, McQuestion M. Putting evidence into practice: evidence-based interventions for radiation dermatitis. Clin J Oncol Nurs. 2011;15:481-92.

13. Salzmann M, Marmé F, Hassel JC. Prophylaxis and management of skin toxicities. Breast Care (Basel). 2019;14:72-7.

14. Guillot B, Bessis D, Dereure O. Mucocutaneous side effects of antineoplastic chemotherapy. Expert Opin Drug Saf. 2004;3:579-87.

15. Huang WY, Hong JB, Chang M, Wang SY, Lai SF, Chein HF, et al. Lower proximal cup and outer root sheath cells regenerate hair bulbs during anagen hair follicle repair after chemotherapeutic injury. Exp Dermatol. 2021;30:503-11.

16. Rebora A, Guarrera M. Why do not all chemotherapy patients lose their hair? Answering an intriguing question. Skin Appendage Disord. 2021;7:280-85.

17. Fabbrocini G, Cameli N, Romano MC, Mariano M, Panariello L, Bianca D, et al. Chemotherapy and skin reactions. J Exp Clin Cancer Res. 2012;31:50.

18. Vigarios E, Epstein JB, Sibaud V. Oral mucosal changes induced by anticancer targeted therapies and immune checkpoint inhibitors. Support Care Cancer. 2017;25:1713-39.

19. Nikolaou V, Syrigos K, Saif MW Incidence and implications of chemotherapy related hand-foot syndrome. Expert Opin Drug Saf. 2016;15:1625-33.

20. Maillot O, Leduc N, Atallah V, Escarmant P, Petit A, Belhomme S, et al. Evaluation of acute skin toxicity of breast radiotherapy using thermography: results of a prospective single-centre trial. Cancer Radiother. 2018;22:205-10.

21. Chan RJ, Larsen E, Chan P. Re-examining the evidence in radiation dermatitis management literature: an overview and a critical appraisal of systematic reviews. Int J Radiat Oncol Biol Phys. 2012;84:e357-62.

Copyright by Abla Sefako Akakpo, et al. This is an open-access article distributed under the terms of the Creative Commons Attribution License, which permits unrestricted use, distribution, and reproduction in any medium, provided the original author and source are credited.

Source of Support: Nil, Conflict of Interest: None declared. 